\title{
Reviewers for Volumes 17 - 19
}

The editors would like to thank the following reviewers for their contribution to Volumes 17 - 19 of Research in Learning Technology:

Tom Abeles

Abdullah Al-Zoubi

Christopher Alexander

Graham Alsop

Kyriaki Anagnostopoulou

Trish Andrews

Inmaculada

Arnedillo-Sánchez

Jill Attewell

Jo Axe

Jason Baker

Phil Barker

Hans-Peter Baumeister

Sian Bayne

Doug Belshaw

Greg Benfield

Liz Bennett

Sue Bennett

Angela Benson

Canan Blake

Ana-Maria Bliuca

Alexandre Borovik

Adele Botha

John Bourne

Paul Brett

Kevin Brosnan

Mark Brown

Stephen Brown

Kevin Burden

Thomas Carey

Tony Carr

Cathy Cavanaugh

Mark Childs

Valerie Clifford

Lynn Clouder

Thomas Cochrane

Grainne Conole
John Cook

Martyn Cooper

Sarah Cornelius

Linda Creanor

Charles Crook

Marija Cubric

Roberta Cuel

Juliette Culver

Laura Czerniewicz

Cristina da Costa

Barney Dalgarno

Inge de Waard

Frances Deepwell

Maarten deLaat

Lesley Diack

Michele Dickey

Roisin Donnelly

EA Draffan

Stephen Draper

Damian Duffy

Charles Dziuban

Martin Ebner

Nigel Ecclesfield

Palitha Edirisingha

Rob Edmunds

Chris Evans

Liz Falconer

Vincent Farrell

Andrew Feenberg

Rebecca Ferguson

Ian Foster

Bob Fox

Randy Garrison

Mark Gaved

Sebastian George

Graham Gibbs

Julia Gillen
Judith Good

Robin Goodfellow

Peter Goodyear

Cathy Gunn

Tak Ha

Laurence Habib

Martin Hall

Richard Hall

Janet Hanson

Peter Hartley

Jen Harvey

Stylianos Hatzipanogos

David Hawkridge

Caroline

Haythornthwaite

John Hedberg

Aleksej Heinze

Cheryl Hodgkinson

Williams

Katherine Howland

Shaheeda Jaffer

Jill Jameson

Ann Jones

Chris Jones

Peter Kahn

James Kariuki Njena

Karen Kear

Matthew Kearney

Helen Keegan

Mike Keppell

Cindy Kerawalla

Hyo Kim

Adrian Kirkwood

Marguerite Koole

Rita Kop

Rob Koper

Mark Kramer 


\begin{tabular}{|c|c|c|}
\hline Agnes Kukulska-Hulme & Meg O'Reilly & Donald Spicer \\
\hline Mark Lee & Martin Oliver & Lorraine Stefani \\
\hline Oleg Liber & Ron Oliver & Ian Stevenson \\
\hline Daniel Livingstone & Norbert Pachler & Brian Stewart \\
\hline Bill Lockitt & Santosh Panda & Lynn Tabata \\
\hline Peter Lonsdale & Marina Papastergiou & Brendan Tangney \\
\hline Jon Loose & Don Passey & Marie Tapanes \\
\hline Betsy Lyon & Valerie Paton & Jenepher Lennox Terrion \\
\hline Janet Macdonald & Elaine Pearson & Michael Thomas \\
\hline Terry Mayes & Vanessa Pfeiffer & Mary Thorpe \\
\hline Brodie McAdam & Rob Phillips & Jo Tomalin \\
\hline Patrick McAndrew & Palmyre Pierroux & Christopher Tompsett \\
\hline Claire McAvinia & Stephen Powell & John Traxler \\
\hline David McConnell & Tom Power & Najim Ussiph \\
\hline Holly McCracken & Andy Pulman & Herman van der Merwe \\
\hline Catherine McLoughlin & Ruslan Ramanau & Giasemi Vavoula \\
\hline Barry McMullin & Rebecca Reynolds & George Veletsianos \\
\hline Carmel McNaught & John Richardson & Pascal Venier \\
\hline Margot McNeill & Carol Russell & Kevin Walker \\
\hline Michael McVey & Malcolm Rutter & Robert Ward \\
\hline Harvey Mellar & Thomas Ryberg & Ng Wee Leng \\
\hline Robbie Melton & Gilly Salmon & Brian Whalley \\
\hline Andrew Middleton & John Sandars & Janice Whatley \\
\hline Marcelo Milrad & Seb Schmoller & Steve Wheeler \\
\hline David Morris & Judith Schoonenboom & Denise Whitelock \\
\hline Andrew Morrison & Jane Seale & Peter Whitton \\
\hline Charalambos Mouzakis & Seyyed Abdollah & Ann Wilkinson \\
\hline Elizabeth Murphy & Shahrokni & Jocasta Williams \\
\hline Daisy Mwanza-Simwami & Mike Sharples & Kevin Williams \\
\hline Som Naidu & Jennifer Sheridan & Gail Wilson \\
\hline Chrissi Nerantze & Vanessa Simonite & Scott Wilson \\
\hline Monika Nerland & Christine Sinclair & Niall Winters \\
\hline Barbara Newland & Peter Sloep & Jocelyn Wishart \\
\hline Mark Nichols & Carl Smith & Iñigo Yanguas \\
\hline Ming Nie & Jan Smith & Patricia Young \\
\hline John O'Donoghue & Marcus Specht & Shenghua Zha \\
\hline
\end{tabular}

As editors, our approach continues to be one of supporting authors in developing and improving their submissions to Research in Learning Technology through a constructive, iterative process. Our reviewers enable us to do this by providing detailed and considered feedback to authors. Collectively, our reviewers have contributed to enhancing the quality of Research in Learning Technology, and we are extremely grateful. 